\title{
Monte Carlo integration in Glauber model analysis of reactions of halo nuclei
}

\author{
K. Varga ${ }^{1 *}$, Steven C. Pieper ${ }^{2 \dagger}$, Y. Suzuki ${ }^{3 \ddagger}$, and R. B. Wiringa ${ }^{2 \S}$ \\ 1 Solid State Division, \\ Oak Ridge National Laboratory, \\ Oak Ridge, 37831 Tennessee, USA \\ and \\ Institute for Nuclear Research of the Hungarian Academy of Sciences (ATOMKI), \\ 4000 Debrecen, Hungary PO BOX 51 \\ 2 Argonne National Laboratory, \\ Argonne, IL 60439, USA \\ 3 Department of Physics, \\ Niigata University, \\ Niigata 950-2181, Japan
}

\begin{abstract}
Reaction and elastic differential cross sections are calculated for light nuclei in the framework of the Glauber theory. The optical phase-shift function is evaluated by Monte Carlo integration. This enables us to use the most accurate wave functions and calculate the phase-shift functions without approximation. Examples of proton nucleus (e.g. p- ${ }^{6} \mathrm{He}, \mathrm{p}-{ }^{6} \mathrm{Li}$ ) and nucleus-nucleus (e.g. ${ }^{6} \mathrm{He}-{ }^{12} \mathrm{C}$ ) scatterings illustrate the effectiveness of the method. This approach gives us a possibility of a more stringent analysis of the high-energy reactions of halo nuclei.
\end{abstract}

PACS numbers:

\footnotetext{
* (e-mail: vargak@ornl.gov).

$\dagger$ (e-mail: spieper@anl.gov)

$\ddagger$ (e-mail: suzuki@nt.sc.niigata-u.ac.jp)

$\S$ (e-mail: wiringa@anl.gov)
} 


\section{INTRODUCTION}

The study of hundreds of new unstable nuclei has become possible in the new radioactive beam facilities. The measurement of the cross sections at high energies is an important experimental tool for studying these elements [1]. The observed interaction cross sections can be related to the wave functions of these nuclei through the Glauber theory [2]; thus one can obtain information on the structure of these exotic isotopes. This direct relation between the structure and reaction cross section is, however, hampered by several problems. The first and most serious one is that the calculation of the cross section by using accurate nuclear wave functions is difficult. Also the solution of the nuclear many-body problem is notoriously complicated.

In Glauber theory the nucleus-nucleus elastic scattering amplitude is obtained by integrating the optical phase-shift function over the impact parameter. The optical phase-shift function is the matrix element of the multiple-scattering operator between the product of wave functions of the target and projectile. The difficulty of the evaluation of this matrix element stems from the fact that the multiple-scattering operator is an $A$-body ( $A$ is the total number of nucleons involved) operator, a product of pairwise nucleon-nucleon scattering operators. To avoid the calculation of the matrix element of an $A$-body operator several simplifying approximations have been introduced over the years. One popular method is to replace the wave function of the target or both the projectile and the target by densities. This is then further simplified by assuming that the density of the nucleus is a product of one-body densities. In that case the matrix element of the multiple-scattering operator becomes a product of two-body matrix elements and is easy to calculate. Another popular approach is to use an inert core and concentrate on the effect of the valence particles only. One can also expand the multiple-scattering operator as a sum of two-, three-, ..., $A$ body operators and truncate the expansion at some level. The most popular method is the "optical-limit approximation" (OLA) where the complicated multiple-scattering operator is replaced by a simple two-body operator in a somewhat ad hoc way.

The problem of these approximations is that as the "exact" scattering amplitude is not available, it is very difficult to judge how good they are. Some of the assumptions sound very serious; the usage of one-body densities most definitely washes out the effects of nuclear correlations. Some of these approximations may be especially bad for weakly bound halo 
nuclei as has been discussed by many authors [3, 4, 5, 6, 7].

To avoid the complication of the analytical calculation of the cross section we will use Monte Carlo integration. We will show that the accuracy of this approach is very good and one can obtain very reliable results with very little effort compared to the previous approaches. Moreover, unlike the previous calculations, no approximation has to be introduced in the evaluation of the Glauber amplitude. We use many-body wave functions directly without loosing delicate correlation effects. In the framework of the Glauber theory, Monte Carlo integration has been used to calculate the nuclear transparency in the $\gamma n \rightarrow \pi^{-} p$ process in ${ }^{4} \mathrm{He}$ and ${ }^{16} \mathrm{O}$ in Ref. [8].

This approach also allows us to use the most advanced quantum Monte Carlo (QMC) wave functions available for light nuclei. These wave functions are obtained by the solution of the nuclear Hamiltonian with realistic two- and three-body interactions. These ab initio QMC calculations of the energy spectra and other nuclear properties are in good agreement with experiments in the $A \leq 8$ region [9]. The QMC predicts the radii of the light nuclei quite reliably so that a direct comparison of the nuclear sizes and reaction cross sections becomes possible.

The road map of this paper is as follows. First we introduce the most important quantities of the Glauber theory in Sec. II. Next we show some of the details of the calculation in Sec. III and of the wave functions in Sec. IV. The presentation of the results can be found in Sec. V. A brief summary and outlook closes the paper in Sec. VI.

\section{GLAUBER THEORY}

Glauber's multiple-scattering theory [2] of high-energy collisions, which is based on the eikonal and adiabatic approximations, is widely accepted as a standard tool for calculating various cross sections. The probability that two colliding nucleons in the projectile and the target lead to the excitation of the nuclei is almost unity in high-energy reactions (at several hundred $\mathrm{MeV} / A$ ). Since the nuclear force is short-ranged, the probability of nuclear excitation in the collision reflects the geometrical size of the nuclei. The interaction or reaction cross section can thus be related to the size and structure of the nuclei.

The basic quantity in the Glauber theory is the optical phase-shift function $\chi_{\mathrm{el}}(\boldsymbol{b})$ defined 
by

$$
\mathrm{e}^{i \chi_{\mathrm{el}}(\boldsymbol{b})}=\left\langle\Phi_{0}^{\mathrm{P}} \Phi_{0}^{\mathrm{T}}\left|\prod_{i \in \mathrm{P}} \prod_{j \in \mathrm{T}}\left[1-\Gamma\left(\boldsymbol{b}+\boldsymbol{s}_{i}^{\mathrm{P}}-\boldsymbol{s}_{j}^{\mathrm{T}}\right)\right]\right| \Phi_{0}^{\mathrm{P}} \Phi_{0}^{\mathrm{T}}\right\rangle,
$$

where $\boldsymbol{b}$ is the impact parameter, and $\Phi_{0}^{\mathrm{P}}\left(\Phi_{0}^{\mathrm{T}}\right)$ is the intrinsic projectile(target) wave function with its center-of-mass wave function removed. The profile function $\Gamma$ is a two-dimensional Fourier transform of the nucleon-nucleon scattering amplitude

$$
f(\theta, \phi)=\frac{i k}{2 \pi} \int d \boldsymbol{b} \mathrm{e}^{-i \boldsymbol{q} \cdot \boldsymbol{b}} \Gamma(\boldsymbol{b}) .
$$

In Eq. (1) one integrates over all (independent) intrinsic coordinates $\boldsymbol{r}_{i}^{\mathrm{P}}$ and $\boldsymbol{r}_{j}^{\mathrm{T}}$. The twodimensional vectors, $\boldsymbol{s}_{i}^{\mathrm{P}}$ and $\boldsymbol{s}_{j}^{\mathrm{T}}$, are the projections of $\boldsymbol{r}_{i}^{\mathrm{P}}$ and $\boldsymbol{r}_{j}^{\mathrm{T}}$ onto the $x y$-plane which is perpendicular to the incident direction of the projectile.

The nucleus-nucleus elastic scattering amplitude is easily obtained once the optical phaseshift function is available:

$$
f_{\mathrm{el}}(\theta, \phi)=\frac{i K}{2 \pi} \int d \boldsymbol{b} \mathrm{e}^{-i \boldsymbol{q} \cdot \boldsymbol{b}}\left[1-\mathrm{e}^{i \chi_{\mathrm{el}}(\boldsymbol{b})}\right],
$$

where $K$ is the wave number of the relative motion between the two nuclei. The effects of the Coulomb interaction can be easily incorporated as well, but, as they are important only at extreme forward angles, we omitted the Coulombic contribution in the present analysis. The total reaction cross section is obtained by subtracting the elastic cross section from the total cross section:

$$
\sigma_{\text {reac }}=\int d \boldsymbol{b}\left(1-\left|\mathrm{e}^{i \chi_{\mathrm{el}}(\boldsymbol{b})}\right|^{2}\right)
$$

The Glauber theory is a non-perturbative theory; its strength is that it directly employs information on the bare nucleon-nucleon interaction and thereby makes it possible to relate the reaction dynamics to the underlying interaction of the constituent particles. At lower energies and for heavier nuclei than those considered in this paper, the bare nucleon-nucleon cross sections must be corrected for medium effects related to Pauli blocking and effective masses [10]. Usually $\Gamma$ is not calculated from the bare nucleon-nucleon interaction but parametrized in a convenient form (like Gaussians) so as to fit the empirical nucleon-nucleon scattering amplitude through Eq. (2):

$$
\Gamma(\boldsymbol{b})=\frac{\sigma_{\tau}}{4 \pi \beta_{\tau}}\left(1-i \alpha_{\tau}\right) \exp \left(-\frac{\boldsymbol{b}^{2}}{2 \beta_{\tau}}\right)
$$


where the parameters depend on the isospin of the nucleons $(\tau=\mathrm{pp}, \mathrm{nn}, \mathrm{pn})$. Other operators, like spin-orbit and tensor, may also be necessary (especially if the spins of the target and projectile are nonzero), but those are most often neglected. Their inclusion would not cause any problem in the present approach.

As seen in Eq. (1), the optical phase-shift function is defined by a many-body multiplescattering operator and obviously its calculation is very involved. One often uses some simplification like the OLA, which is just the first-order approximation in the cumulant expansion [2]:

$$
\mathrm{e}^{i \chi_{\mathrm{OLA}}(\boldsymbol{b})}=\exp \left\{-\iint d \boldsymbol{r}^{\mathrm{P}} d \boldsymbol{r}^{\mathrm{T}} \rho^{\mathrm{P}}\left(\boldsymbol{r}^{\mathrm{P}}\right) \rho^{\mathrm{T}}\left(\boldsymbol{r}^{\mathrm{T}}\right) \Gamma\left(\boldsymbol{s}^{\mathrm{P}}-\boldsymbol{s}^{\mathrm{T}}+\boldsymbol{b}\right)\right\},
$$

where e.g., $\rho^{\mathrm{P}}\left(\boldsymbol{r}^{\mathrm{P}}\right)$ is the single-particle density of the projectile nucleus.

Several authors have shown that a treatment beyond the OLA is necessary for a quantitative analysis of the reaction cross sections [3, [4, 5] as well as the elastic scattering cross sections [6, 7]. This is particularly true for loosely coupled systems such as halo nuclei because breakup effects are not properly accounted for in the OLA. There have been considerable efforts which attempt to calculate higher-order or all-order terms of the multiple-scattering operator. It is known that the matrix elements of higher-order terms should be evaluated by two- and more-particle densities [2]. Using the density constructed from uncorrelated wave functions [11, 12, 13, 14] is thus not sufficient to understand the importance of the correlated motion in nuclei even if all-order terms are evaluated.

Very recently a method of calculating the complete Glauber amplitude [15 has been proposed and applied to $\mathrm{p}+{ }^{6} \mathrm{He}$ elastic scattering using a microscopic $\alpha+\mathrm{n}+\mathrm{n}$ three-cluster wave function for ${ }^{6} \mathrm{He}$ [16]. It uses an expansion of the multiple-scattering operator and evaluates each term analytically provided that the profile function $\Gamma$ is expressed by Gaussians. The method has been extended to a nucleus-nucleus case as well with considerable success [17]. However, this method is limited by two things: one is that the number of terms in the expansion becomes prohibitively large for heavier nuclei; another is that if $\Gamma$ is not a Gaussian then the analytic integration for correlated wave functions is hopeless. 


\section{MONTE CARLO INTEGRATION}

Our purpose is to present a powerful method of calculating the matrix element of the multiple-scattering operator completely by taking another route, a Monte Carlo integration (MCI) of the multiple-scattering operator. No method has so far been available to calculate the Glauber amplitude completely for general correlated wave functions. The Monte Carlo integration seems to be the most natural way to calculate the phase-shift function. Its advantages are quite clear: (1) there is no restriction on the form of the target or projectile wave functions (general few- or many-body wave function can be used); (2) the full multiplescattering operator can be used (there is no need for expansion or truncation); (3) it is very simple compared to earlier calculations.

In our present approach the multiple-scattering operator is assumed to be independent of spins, depending only on the spatial coordinates and the isospins. The isospin dependence arises when different profile functions are used between the pp (nn) and pn pairs. The wave functions $\Phi_{0}^{\mathrm{P}}$ and $\Phi_{0}^{\mathrm{T}}$ are represented by a multicomponent (approximately $2^{A} \times\left({ }_{Z}^{A}\right)$ ) vector in the spin-isospin space. The matrix elements are calculated by taking the scalar product of the bra and the ket in the spin-isospin space and using MCI in the coordinate space.

In MCI, the integration points are generated by the Metropolis algorithm using $\left|\Phi_{0}^{\mathrm{P}} \Phi_{0}^{\mathrm{T}}\right|^{2}$ as a guiding function. One may possibly get better accuracy and convergence by using $\left(a+b r^{2}\right)\left|\Phi_{0}^{\mathrm{P}} \Phi_{0}^{\mathrm{T}}\right|^{2}$ ( $r$ is the root mean square radius of the projectile) or some similar expression which increases the weight of the asymptotic part, but as will be shown later the simple $\left|\Phi_{0}^{\mathrm{P}} \Phi_{0}^{\mathrm{T}}\right|^{2}$ form is sufficient for the present purposes.

In the MCI we first generate a set of $N$ integration points by a Metropolis random walk and store them. Then the optical phase-shift function is calculated over these configurations for each discretized value of the impact parameter $b$. In this way we not only save computational time but we have a "correlated sampling" for different impact parameters avoiding the independent statistical errors of multiple Metropolis walks. The reaction cross section or the elastic differential cross section is calculated by numerical integration over the impact parameter.

The impact parameter $\boldsymbol{b}$ is a two-dimensional vector which can be parametrized as $(b \sin \phi, b \cos \phi)$. For spherical nuclei the phase-shift function has no dependence on $\phi$, so the integration in Eqs. (3) and (4) over $\boldsymbol{b}$ can be reduced to that over the radial variable $b$. 
In the nonspherical case, one has a two-dimensional integration over the impact parameter, which increases the number of discretization points. The practical example studied in this paper shows that the dependence on $\phi$ is almost negligible.

To test the MCI evaluation of the optical phase-shift function, a simple but nontrivial example, $\alpha+\alpha$ scattering at $5.07 \mathrm{GeV} / c\left(T_{\alpha}=2.57 \mathrm{GeV}\right)$, has been considered. Taking a single harmonic-oscillator shell-model wave function for the $\alpha$-particle, the phase-shift function and the reaction cross section can be analytically calculated [15]. The MCI results are tested against this analytical example. Figure 1 shows the elastic differential cross section as a function of four-momentum transfer squared $-t=\hbar^{2} \boldsymbol{q}^{2}$ using 100,000 MCI points. For small momentum transfer (this is the region where the present day experiments can be performed) a very small number of MCI points is sufficient to get reliable results. For larger momenta the oscillatory behavior coming from $\mathrm{e}^{-i \boldsymbol{q} \cdot \boldsymbol{b}}$ becomes more rapid, requiring more accurate integration for relatively large $b$, but even for the $10^{5}$ points used the computational time is still almost negligibly small. Table I shows the reaction cross section and the mean-square (rms) radius of the $\alpha$-particle obtained from this calculation. Naturally, the analytically calculated values are perfectly reproduced provided one uses a sufficiently large number of MCI configurations. These tests show that one can confidently use the MCI in calculation of the optical phase-shift function and related quantities.

\section{WAVE FUNCTIONS}

The wave functions, except for ${ }^{12} \mathrm{C}$, are obtained by the QMC method [9, 18] using the Argonne $v_{18}$ two-body [19] and the Illinois three-body interaction IL2 [20]. The QMC method with these interactions provides a reliable description of the energy levels and different properties of light nuclei. Previous calculations are based on cluster, few-body, shell-model or mean-field wave functions to calculate the optical phase-shift functions. These calculations have used schematic effective interactions with adjustable parameters and other simplifying approximations. The use of QMC provides us with a realistic, ab initio, microscopic wave function. The ${ }^{12} \mathrm{C}$ nucleus is not yet accessible in QMC and for the ${ }^{12} \mathrm{C}$ a three- $\alpha$ microscopic cluster-model wave function is used. In this model the internal wave functions of the $\alpha$ particles are single shell-model Slater determinants and the relative motion between the clusters is represented by linear combinations of Gaussians. The combination coefficients 
are determined variationally by solving the 12-nucleon Schrödinger equation with an effective (Minnesota [21]) two-nucleon interaction.

The QMC methods include variational Monte Carlo (VMC) and Green's function Monte Carlo (GFMC) methods. The VMC is an approximate method that is used as a starting point for the more accurate GFMC calculations. The VMC method starts with the construction of a variational trial function of specified angular momentum, parity and isospin, $\Psi_{T}\left(J^{\pi} ; T\right)$, using products of two- and three-body correlation operators acting on a fully antisymmetrized set of one-body basis states. Metropolis Monte Carlo integration is used to evaluate $\left\langle\Psi_{T}|H| \Psi_{T}\right\rangle$, giving an upper bound to the energy of the state. The GFMC method is a stochastic method that systematically improves on $\Psi_{T}$ by projecting out excited state contamination using the Euclidean propagation $\Psi(\tau)=\exp \left[-\left(H-E_{0}\right) \tau\right] \Psi_{T}$. In the limit $\tau \rightarrow \infty$, this leads to the exact ground state energy. Details of the structure calculations can be found in [9].

The calculation of expectation values directly using

$$
\langle O(\tau)\rangle=\frac{\langle\Psi(\tau)|O| \Psi(\tau)\rangle}{\langle\Psi(\tau) \mid \Psi(\tau)\rangle}
$$

is complicated and computationally too demanding. In our calculations we have used the approximate expression:

$$
\langle O(\tau)\rangle \approx\langle O(\tau)\rangle_{\text {Mixed }}+\left[\langle O(\tau)\rangle_{\text {Mixed }}-\langle O\rangle_{T}\right]
$$

where the "mixed" expectation value between $\Psi_{T}$ and $\Psi(\tau)$ is:

$$
\langle O\rangle_{\text {Mixed }}=\frac{\left\langle\Psi_{T}|O| \Psi(\tau)\right\rangle}{\left\langle\Psi_{T} \mid \Psi(\tau)\right\rangle}
$$

and $\langle O\rangle_{T}$ is just the variational expectation value. This approximation is very good, provided that the difference between the VMC trial function $\Psi_{T}$ and the exact wave function is reasonably small.

\section{RESULTS}

In this section we present our results for total reaction and elastic differential cross sections of different projectile-target systems. The proton, neutron and matter rms radii of the nuclei predicted by the structure calculations are shown in Table II. The VMC gives a very good 
wave function for the alpha particle, but underbinds the $A=6$ nuclei. The GFMC energies of ${ }^{6} \mathrm{He}$ and ${ }^{6} \mathrm{Li}$ are in very good agreement with experiment. The GFMC improvement of the wave function is especially important for the loosely bound ${ }^{6} \mathrm{He}$.

As a first application we have calculated the differential cross section for $\mathrm{p}+\alpha$ elastic scattering at $T_{\mathrm{p}}=0.7 \mathrm{GeV}$ (see Fig. 2). (The parameters of the profile function (5) are taken from [15]). The agreement with the observed cross section [22, 23] is perfect, but unfortunately the experimental data are only available up to $0.05(\mathrm{GeV} / \mathrm{c})^{2}$. The differential cross section obtained by a simple harmonic-oscillator $\alpha$-particle wave function is also included for comparison. The realistic and simple shell-model prediction is nearly identical in the experimentally accessible region. For larger momentum transfer the two wave functions predict significantly different cross sections despite the fact that they give the same nuclear radius.

Next we consider the $\alpha+\alpha$ elastic scattering at $5.07 \mathrm{GeV} / c$. In this case the experimental data 24] are available for a wider range of momentum transfer (see Fig. 3). While the simple shell-model wave function fails to explain the observed data points, the realistic wave function leads to good agreement with the experiments. The disagreement between the simple shell-model and realistic wave function prediction is almost an order of magnitude. To explain the data with a simple shell-model wave function one has to increase the harmonic-oscillator size parameter drastically and that would lead to an unrealistic $\alpha$ particle radius (about 20\% too large). This example clearly shows the importance of the realistic wave functions and the sensitivity of the experimental results to the details of the nuclear structure.

Table III compares the reaction cross sections calculated for various systems with measured interaction cross sections, $\sigma_{\text {int }}$. The interaction cross section is defined as the sum of the cross sections for all channels in which the nucleonic composition of the projectile changes. In high-energy collisions, the projectile can only lose nucleons, that is, the probability of pickup processes is negligible, and the difference between $\sigma_{\text {reac }}$ and $\sigma_{\text {int }}$ is expected to be small. Possible differences between them come from two processes: one is the inelastic excitation of the projectile, which may occur if the projectile has a particle-bound excited state. Another is a process in which the projectile remains in its ground state while the target gets excited. These processes contribute to $\sigma_{\text {reac }}$ but not to $\sigma_{\text {int }}$. When the projectile is a halo nucleus that has no particle-bound state, like ${ }^{6} \mathrm{He}$, the second process can be ignored because the halo nucleus is easily broken by a small shock so it is unlikely that it remains in 
its ground state while exciting the target. The example of ${ }^{6} \mathrm{He}+{ }^{12} \mathrm{C}$ collision at $0.8 \mathrm{GeV} / A$ bears out this argument; the calculated reaction cross section is indeed close to the measured interaction cross section. The ${ }^{6} \mathrm{Li}+{ }^{12} \mathrm{C}$ reaction cross section is slightly larger than the interaction cross section. It remains an open question whether this difference can be accounted for by the above processes. A similar comment may be applied to the ${ }^{12} \mathrm{C}+{ }^{12} \mathrm{C}$ case. For the sake of reference the reaction cross sections measured at $0.87 \mathrm{GeV} / A$ are included in the table.

Figures 4.a and 4.b present the elastic differential cross sections for $\mathrm{p}+{ }^{6} \mathrm{He}$ and $\mathrm{p}+{ }^{6} \mathrm{Li}$ at $T_{\mathrm{p}}=0.7 \mathrm{GeV}$. The experimental data [23, 25] are available only up to $-t=0.05(\mathrm{GeV} / c)^{2}$. In that region (Fig. 5.b) the best theoretical (GFMC) cross sections slightly underestimate the experimental data, especially for ${ }^{6} \mathrm{He}$. An $\alpha+\mathrm{n}+\mathrm{n}$ cluster-model result [15] for $\mathrm{p}+{ }^{6} \mathrm{He}$ at $T_{\mathrm{p}}=0.717 \mathrm{GeV}$, which is obtained by using the wave function 16 solved in a restricted model space with the Minnesota effective interaction, is also included for comparison. The cluster-model result agrees perfectly with the experimental data. (See Fig. 2 of Ref. [15].) The matter rms radius $2.51 \mathrm{fm}$ in the cluster model, $2.56 \mathrm{fm}$ in the VMC and 2.61 in the GFMC calculation. The slight underestimation of the differential cross section by the GFMC might be due to the fact that the size of ${ }^{6} \mathrm{He}$ given by GFMC is a little too large. For higher momentum transfer, there is a substantial difference between ${ }^{6} \mathrm{He}$ and ${ }^{6} \mathrm{Li}$ as well as the cluster and QMC results.

The full and OLA calculations are compared for the reaction and elastic differential cross sections. Table III compares the reaction cross section for $\mathrm{p}+{ }^{6} \mathrm{He}$ and $\mathrm{p}+{ }^{6} \mathrm{Li}$ at $T_{\mathrm{p}}=0.7 \mathrm{GeV}$. The OLA cross section is slightly smaller than that of the full calculation, which, differently

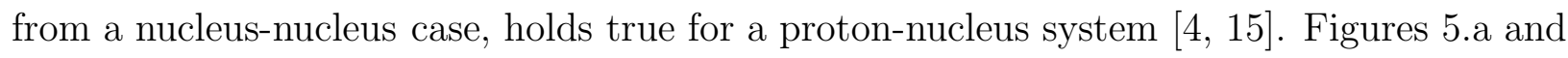
5.b compare the elastic differential cross sections of $\mathrm{p}+{ }^{6} \mathrm{He}$ and $\mathrm{p}+{ }^{6} \mathrm{Li}$ at $T_{\mathrm{p}}=0.7 \mathrm{GeV}$. The difference in the cross sections at small four-momentum transfer between the full and OLA calculations is magnified in Fig. 5.b. Some difference can be seen in the cross section versus $-t$ slope for the case of ${ }^{6} \mathrm{He}$ or in the magnitude of the cross section for the case of ${ }^{6} \mathrm{Li}$ at small $|t|$, but the more conspicuous differences appear near and beyond the first dip of the cross section.

Differential cross sections versus four-momentum transfer squared are plotted in Fig. 6 for elastic scattering of several nuclei on a ${ }^{12} \mathrm{C}$ target at $0.8 \mathrm{GeV} / A$ incident energy. The angular distributions show Fraunhofer-type diffraction patterns and the first dips move to smaller 
angles with increasing mass number. The basic feature of these trends can be understood in a strong absorption model (SAM), which is quite reasonable for high-energy collisions of nuclei. In an extreme version of the SAM, the phase-shift function is assumed to satisfy the relation

$$
\mathrm{e}^{i \chi_{\mathrm{el}}(\boldsymbol{b})}=\Theta(b-R) \equiv\left\{\begin{array}{cc}
0 & b<R \\
1 & b \geq R
\end{array}\right.
$$

where $R$ is a cut-off radius, and is on the order of the sum of the radii of the two colliding nuclei. (In reality $\mathrm{e}^{i \chi_{\mathrm{el}}(\boldsymbol{b})}$ has a smooth cut-off distribution.) In the SAM the incoming flux corresponding to the collision with $b<R$ is completely absorbed, while the collision with $b>R$ receives no effect. (Note that the Coulomb interaction is ignored.) Substitution of Eq. (10) into Eq. (3) leads to the scattering amplitude of $f_{\mathrm{el}}(\theta, \phi) \propto J_{1}(q R) / q R$. The differential cross section $\left|f_{\mathrm{el}}(\theta, \phi)\right|^{2}$ thus vanishes at the zeros of the Bessel function $J_{1}(q R)$, whose zeros occur at $q R \approx 3.83,7.02, \ldots$ With increasing $A, R$ in general increases, thus the first zero appears at smaller values of $-t$. The value of $R$ extracted from the dip in the figure corresponds to the empirical radius rather well. The ${ }^{6} \mathrm{He}$ and ${ }^{12} \mathrm{C}$ case does not follow this rule as the size of ${ }^{6} \mathrm{He}$ is larger than that of ${ }^{12} \mathrm{C}$. This is mainly due to the fact that the sharp cut-off assumption is not good for ${ }^{6} \mathrm{He}$ because of its halo structure. The momentum transfer squared corresponding to the second dip is predicted as $(7.02 / 3.83)^{2} \approx 3.4$ times that of the first dip in the sharp cut-off model.

\section{SUMMARY AND OUTLOOK}

The Monte Carlo integration was used to facilitate the evaluation of the complete Glauber amplitude involving the multiple-scattering operator. There was no need to introduce any ad hoc approximation or assumption in this approach. The great advantage of this method is that it enables us to use accurate, sophisticated wave functions of projectile and target nuclei. A number of calculations confirmed that it is possible to directly relate the wave functions to reaction cross sections measured at high energy.

The calculations presented here focused on light nuclei. One can carry out similar investigations for heavier elements provided that suitable wave functions are available. One very often replaces the wave function of the target nucleus with a density distribution. This approximation renders calculations for heavier targets $\left({ }^{27} \mathrm{Al}\right.$ or $\left.{ }^{208} \mathrm{~Pb}\right)$ accessible. In that case 
one has to construct a reliable nucleon-target profile function to replace the nucleon-nucleon profile function, but the rest of the calculation is the same as presented in this work. One further possible approximation is to use an inert core with correlated valence nucleons to construct the wave function of the projectile. Such a step might be necessary to investigate the cross sections of oxygen or sodium isotopes, for example.

The experimental results mostly cover the low-momentum transfer region where the elastic differential cross section is not too sensitive to the details of the wave functions and simple models do quite well. We have shown, however, that there is a strong discrepancy between the predictions of simple model and realistic wave functions. The high-energy reactions therefore may give information on the details of the nuclear structure. For example, the $\alpha+\alpha$ scattering at $5.07 \mathrm{GeV} / \mathrm{c}$, where the experimental data is available for larger momentum transfers, can only be described by using realistic wave functions for the alpha particle. One hopes that the new radioactive beam facilities will provide us experimental data at higher momentum transfer and further tests of the wave functions will become possible.

\section{Acknowledgments}

The work of K.V. is sponsored by the U.S. Department of Energy under contract DEAC05-00OR22725 with the Oak Ridge National Laboratory, managed by UT-Battelle, LLC, and OTKA grant No. T029003 (Hungary). This work was started while K.V. stayed at Niigata in the summer of 2001 as a fellow of Yamada Science Foundation. The work of S.C.P. and R.B.W. is supported by the U.S. Department of Energy, Nuclear Physics Division, under contract No. W-31-109-ENG-38. The work of Y.S. is supported by a Grant-in Aid for Scientific Research (No. 14540249).

[1] I. Tanihata et al., Phys. Rev. Lett. 55, 2676 (1985); Phys. Lett. B160, 380 (1985).

[2] R. J. Glauber, in Lectures on Theoretical Physics, edited by W. E. Brittin and L. C. Dunham (Interscience, New York, 1959), Vol.1, p.315.

[3] G. F. Bertsch, H. Esbensen and A. Sustich, Phys. Rev. C42, 758 (1990).

[4] Y. Ogawa, K. Yabana and Y. Suzuki, Nucl. Phys. A543, 722 (1992); ibid. A539, 295 (1992). 
[5] J. S. Al-Khalili and J. A. Tostevin, Phys. Rev. Lett. 76, 3903 (1996), J. S. Al-Khalili, J. A. Tostevin and I. J. Thompson, Phys. Rev. C54, 1843 (1996).

[6] K. Yabana, Y. Ogawa and Y. Suzuki, Phys. Rev. C45, 2909 (1992).

[7] J. S. Al-Khalili, J. A. Tostevin and J. M. Brooke, Phys. Rev. C55, R1018 (1997).

[8] H. Gao, R. J. Holt, and V. R. Pandharipande, Phys. Rev. C 54, 2779-2782 (1996).

[9] S. C. Pieper and R. B. Wiringa, Annu. Rev. Nucl. Part. Sci. 51, 53 (2001).

[10] V. R. Pandharipande and S. C. Pieper, Phys. Rev. C 45, 791 (1992).

[11] W. Czyz and L. C. Maximon, Ann. Phys. (N.Y.) 52, 59 (1969).

[12] V. Franco and G. K. Varma, Phys. Rev. C18, 349 (1978), G.K. Varma, Nucl. Phys. A294, 465 (1978).

[13] Yin Yichun, Tan Zhenqiang and Chen Kezhong, Nucl. Phys. A440, 685 (1985).

[14] M. M. H. El-Gogary, A. S. Shalaby and M. Y. M. Hassan, Phys. Rev. C58, 3513 (1998).

[15] B. Abu-Ibrahim, K. Fujimura and Y. Suzuki, Nucl. Phys. A657, 391 (1999).

[16] K. Arai, Y. Suzuki and K. Varga, Phys. Rev. C51, 2488 (1995).

[17] B. Abu-Ibrahim and Y. Suzuki, Phys. Rev. C61, 051601 (2000); ibid. C62, 034608 (2000).

[18] B. S. Pudliner, V. R. Pandharipande, J. Carlson, S. C. Pieper and R. B. Wiringa, Phys. Rev. C 56, 1720 (1997).

[19] R. B. Wiringa, V. G. J. Stocks. and R. Schiavilla, Phys. Rev. C 51, 38 (1995).

[20] S. C. Pieper, V. R. Pandharipande, R. B. Wiringa, and J. Carlson, Phys. Rev. C 64, 014001 (2001).

[21] D. R. Thompson, M. LeMere and Y. C. Tang, Nucl. Phys. A286, 53 (1977).

[22] O. G. Grebenjuk, A. V. Khanadeev, G. A. Korolev, S. I. Manayenkov, J. Saudinos, G. N. Velichko and A. A. Voronchev, Nucl. Phys. A500, 637 (1989).

[23] G. D. Alkhazov et al., Phys. Rev. Lett. 78, 2313 (1997).

[24] J. Berger et al., Nucl. Phys. A338, 421 (1980).

[25] P. Egelhof, Prog. Part. Nucl. Phys. 46, 307 (2001).

[26] A. Ozawa, T. Suzuki and I. Tanihata, Nucl. Phys. A693, 32 (2001).

[27] J. Jaros et al., Phys. Rev. C18, 2273 (1978). 
TABLE I: Comparison of $\alpha+\alpha$ reaction cross section at $5.07 \mathrm{GeV} / c$ and the $\mathrm{rms}$ radius of the $\alpha$-particle calculated analytically and by Monte Carlo integration with 100,000 points. The wave function of the $\alpha$-particle is taken as the simple $(0 \mathrm{~s})^{4}$ Slater determinant. The statistical error of the Monte Carlo integration is given in parenthesis.

\begin{tabular}{lll} 
Method & $\sigma_{\text {reac }}(\mathrm{mb})$ & $\left\langle r^{2}\right\rangle\left(\mathrm{fm}^{2}\right)$ \\
\hline analytic & 242.91 & 2.250 \\
MCI $\quad 242.8(7)$ & $2.251(3)$
\end{tabular}

TABLE II: Point proton, neutron and matter rms radii and reaction cross sections for the collision with a $0.7 \mathrm{GeV}$ proton. The Monte Carlo statistical errors for $\sigma_{\text {reac }}$ are $1 \mathrm{mb}$ in all cases.

\begin{tabular}{|c|c|c|c|c|c|}
\hline System & wave function & $r_{\mathrm{p}}(\mathrm{fm})$ & $r_{\mathrm{n}}(\mathrm{fm})$ & $r_{\mathrm{m}}(\mathrm{fm})$ & $\sigma_{\text {reac }}(\mathrm{mb})$ \\
\hline$\alpha$ & $\mathrm{VMC}$ & 1.46 & 1.46 & 1.46 & 100 \\
\hline${ }^{6} \mathrm{He}$ & $\mathrm{VMC}$ & 1.96 & 2.81 & 2.56 & 163 \\
\hline${ }^{6} \mathrm{He}$ & GFMC & 1.92 & 2.87 & 2.61 & 172 \\
\hline${ }^{6} \mathrm{Li}$ & VMC & 2.47 & 2.47 & 2.47 & 165 \\
\hline${ }^{6} \mathrm{Li}$ & GFMC & 2.47 & 2.47 & 2.47 & 166 \\
\hline${ }^{12} \mathrm{C}$ & cluster & 2.36 & 2.36 & 2.36 & 254 \\
\hline
\end{tabular}


TABLE III: Calculated reaction cross sections. Experimental data are the interaction cross sections taken from [26]. Cross sections with ${ }^{\dagger}$ are the reaction cross sections at $0.87 \mathrm{GeV} / A$ 27]. The statistical error of the Monte Carlo integration is given in parenthesis. The reaction cross section marked by $*$ are obtained in the OLA case.

\begin{tabular}{llll} 
System & Energy $(\mathrm{GeV} / A)$ & $\sigma_{\text {reac }}(\mathrm{mb})$ & $\sigma_{\text {int }}(\mathrm{mb})($ Exp. $)$ \\
\hline $\mathrm{p}+\alpha$ & 0.7 & $100(1)$ & \\
$\mathrm{p}+{ }^{6} \mathrm{He}$ & 0.7 & $172(1)$ & \\
$\mathrm{p}+{ }^{6} \mathrm{Li}$ & 0.7 & $166(1)$ & \\
$\mathrm{p}+{ }^{6} \mathrm{He}$ & 0.7 & $166^{*}$ & \\
$\mathrm{p}+{ }^{6} \mathrm{Li}$ & 0.7 & $164^{*}$ & \\
${ }^{6} \mathrm{He}+{ }^{12} \mathrm{C}$ & 0.7 & $721(2)$ & \\
${ }^{6} \mathrm{Li}+{ }^{12} \mathrm{C}$ & 0.7 & $708(2)$ & \\
$\alpha+\alpha$ & 0.6425 & $235(1)$ & \\
$\mathrm{p}+{ }^{12} \mathrm{C}$ & 0.8 & $261(1)$ & $262 \pm 13.5^{\dagger}$ \\
$\alpha+{ }^{12} \mathrm{C}$ & 0.8 & $506(1)$ & $503 \pm 5,527 \pm 26^{\dagger}$ \\
$\mathrm{p}+{ }^{6} \mathrm{He}$ & 0.8 & $178(1)$ & \\
$\mathrm{p}+{ }^{6} \mathrm{Li}$ & 0.8 & $171(1)$ & \\
${ }^{6} \mathrm{He}+{ }^{12} \mathrm{C}$ & 0.8 & $733(2)$ & $722 \pm 6$ \\
${ }^{6} \mathrm{Li}+{ }^{12} \mathrm{C}$ & 0.8 & $712(2)$ & $688 \pm 10$ \\
${ }^{12} \mathrm{C}+{ }^{12} \mathrm{C}$ & 0.8 & $865(1)$ & $856 \pm 9,939 \pm 49^{\dagger}$ \\
& & & \\
& & &
\end{tabular}


FIG. 1: Center-of-mass differential cross section versus four-momentum transfer squared for $\alpha+\alpha$ elastic scattering at $5.07 \mathrm{GeV} / c$. The solid curve is the result of the analytical calculation, while the result of Monte Carlo integration is shown for 100,000 points.

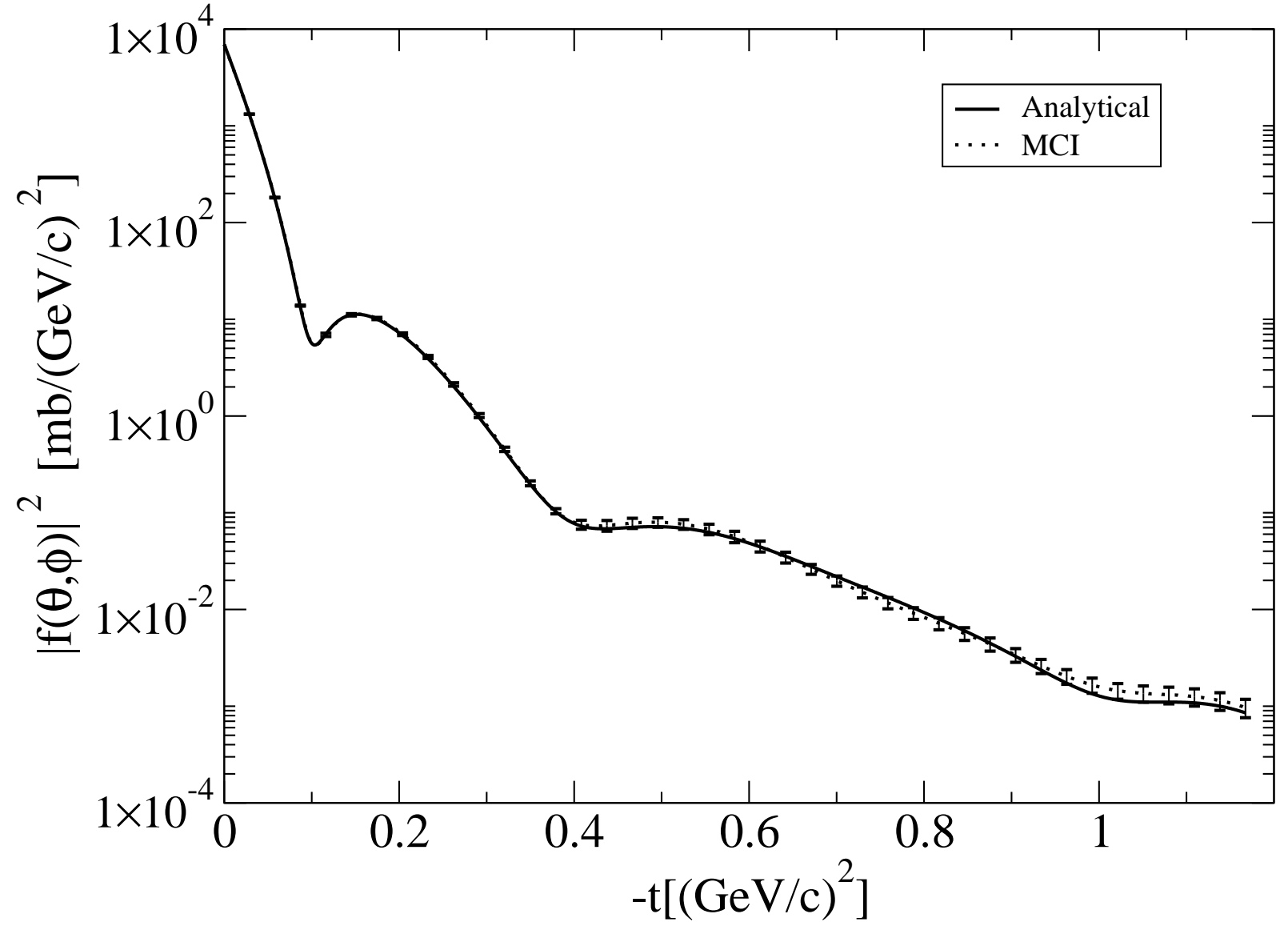




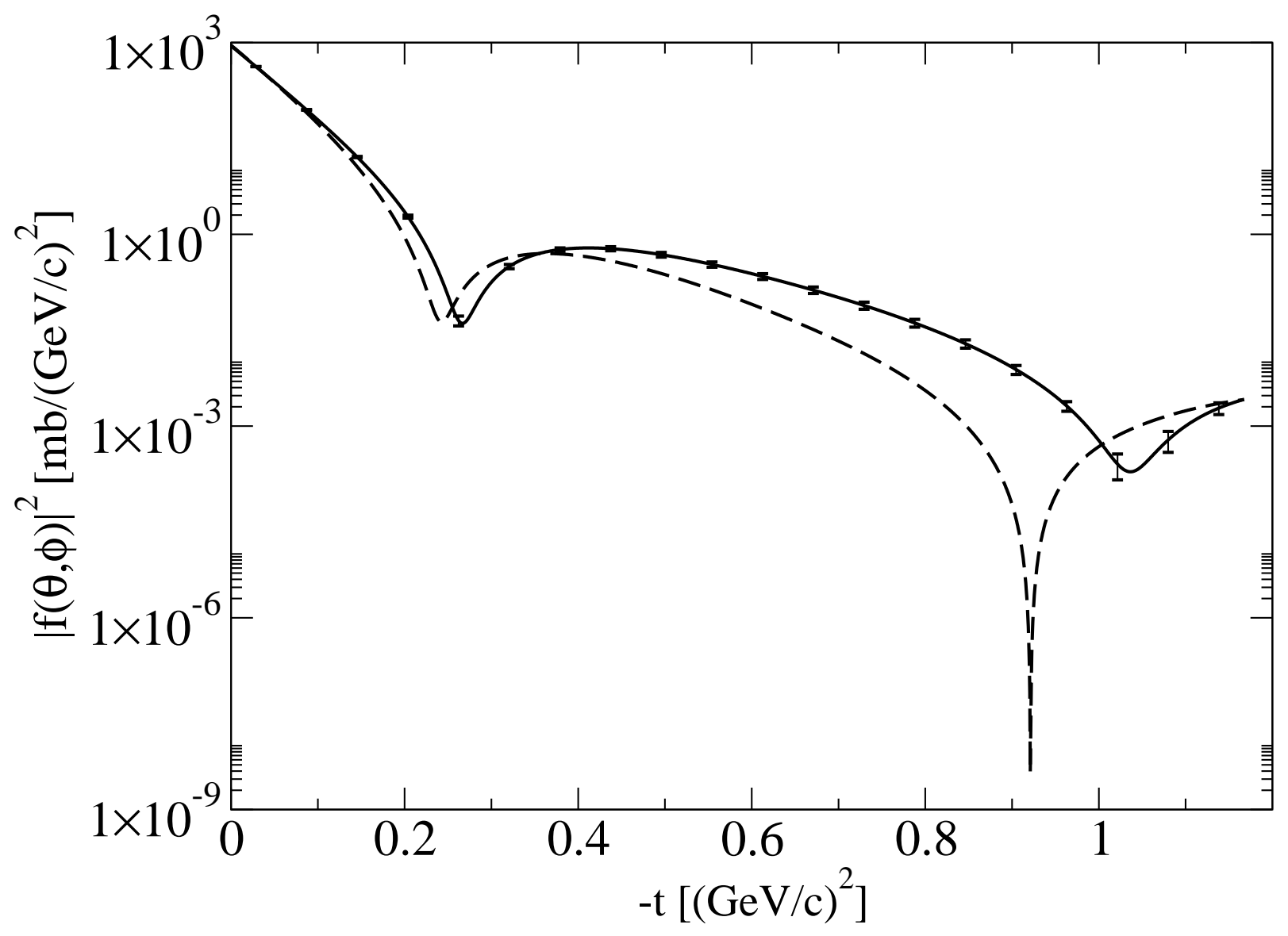

FIG. 2: Comparison of the differential cross section for $\mathrm{p}+\alpha$ elastic scattering at $T_{\mathrm{p}}=0.7 \mathrm{GeV}$ obtained by a simple shell-model (dashed line) and a realistic (solid line) wave function of the $\alpha$-particle. The two wave functions give the same rms radius; Monte Carlo error estimates are shown for the latter case. The data are available only for $-t \leq 0.05(\mathrm{GeV} / c)^{2}$ [22, 23], but not shown because they are hardly distinguishable from the theoretical curves. 


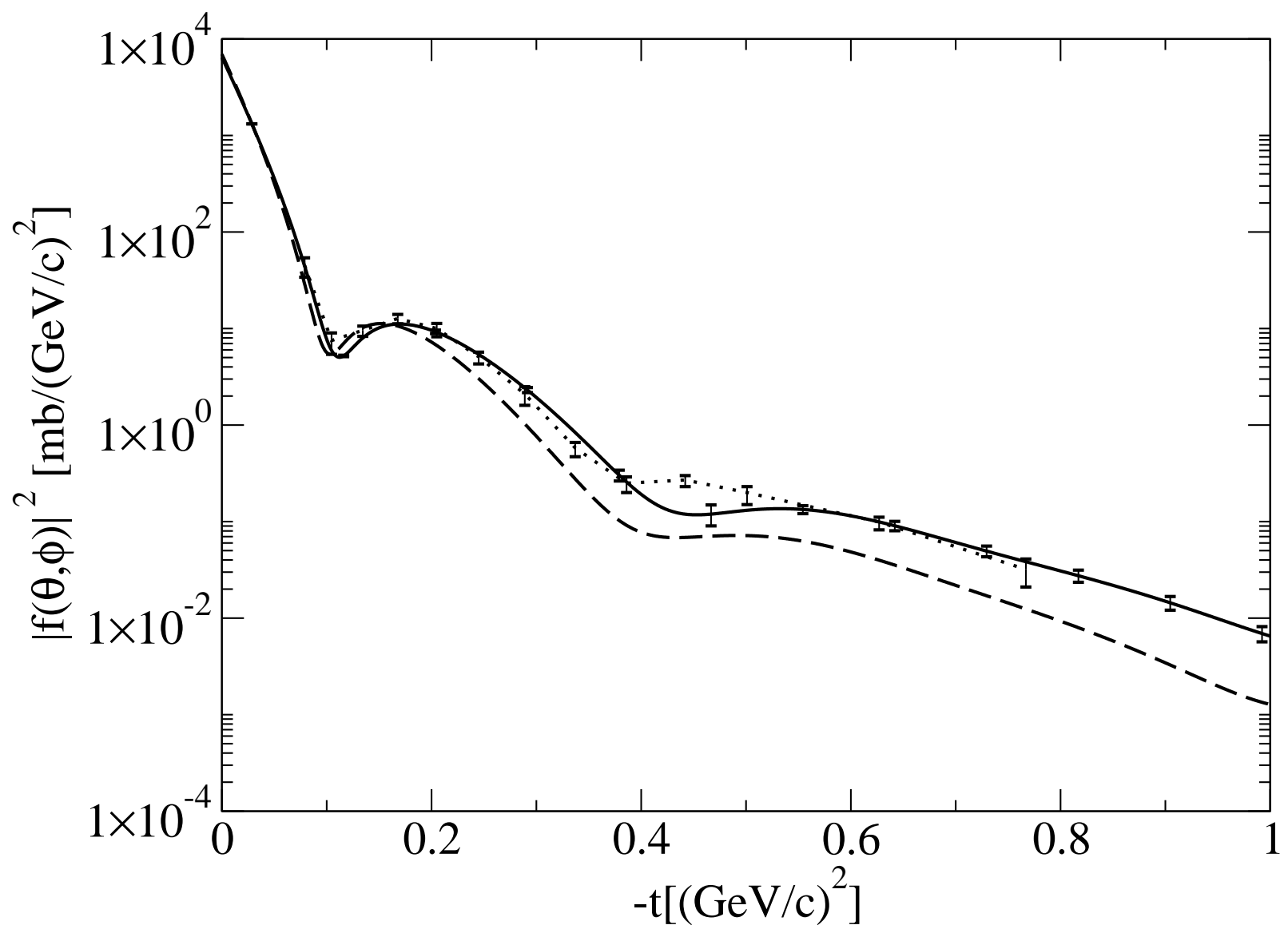

FIG. 3: Comparison of the experimental and theoretical differential cross section for $\alpha+\alpha$ elastic scattering at $5.07 \mathrm{GeV} / c$. The solid line shows the results with a realistic $\alpha$-particle wave function, while the dashed line is obtained by a simple $0 s$ shell-model wave function. The two wave functions give the same rms radius. The data are taken from [24]. 

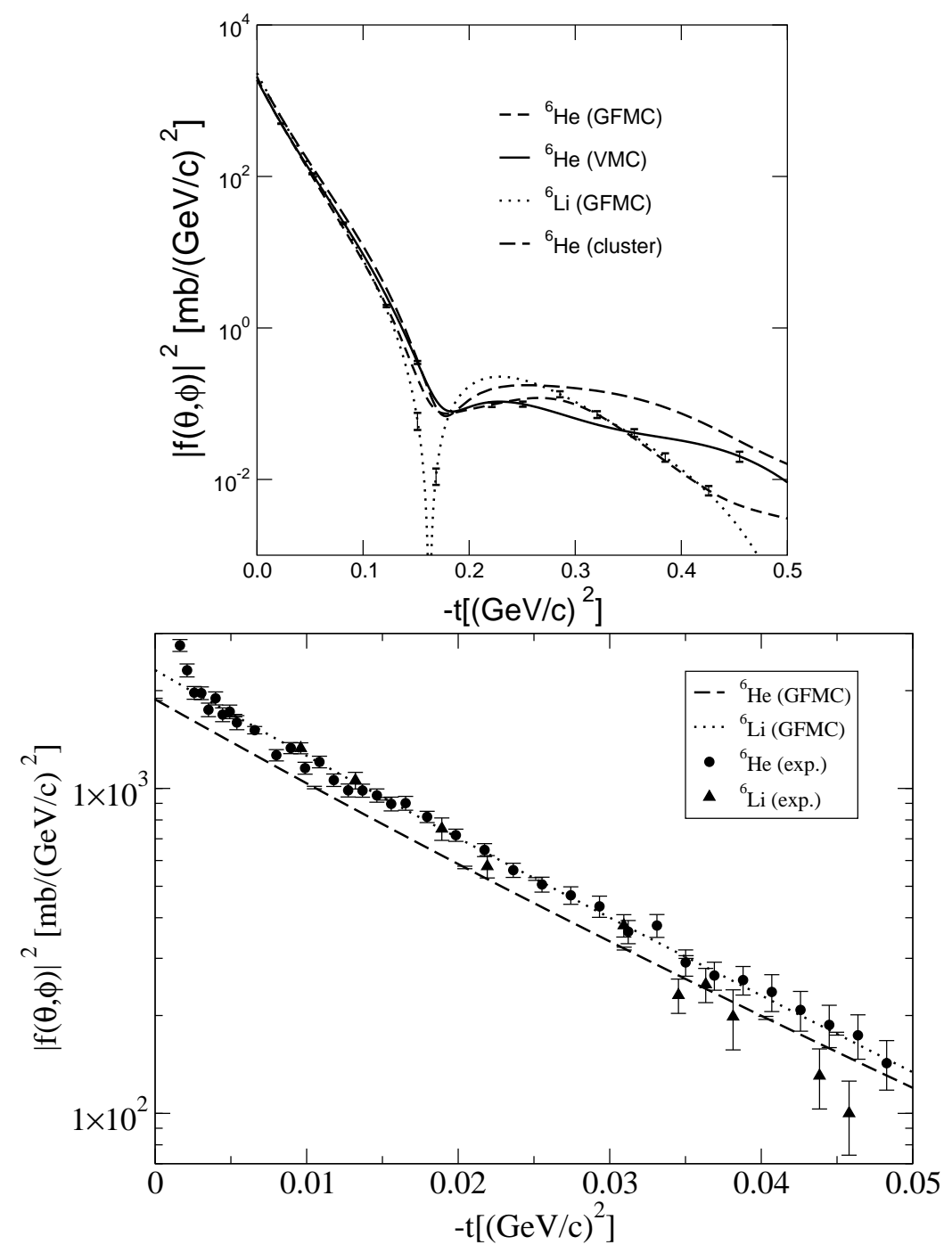

FIG. 4: (a) Differential cross section versus four-momentum transfer squared for $\mathrm{p}+{ }^{6} \mathrm{He}$ and $\mathrm{p}+{ }^{6} \mathrm{Li}$ elastic scatterings at $T_{\mathrm{p}}=0.7 \mathrm{GeV}$. An $\alpha+\mathrm{n}+\mathrm{n}$ cluster-model result 15 is shown for $\mathrm{p}+{ }^{6} \mathrm{He}$ at $T_{\mathrm{p}}=0.717 \mathrm{GeV}$. The statistical error of the Monte Carlo integration is shown for the GFMC and VMC results. (b) Theoretical and experimental [25] differential cross sections for $\mathrm{p}+{ }^{6} \mathrm{He}$ and $\mathrm{p}+{ }^{6} \mathrm{Li}$ elastic scatterings at $T_{\mathrm{p}}=0.7 \mathrm{GeV}$. 

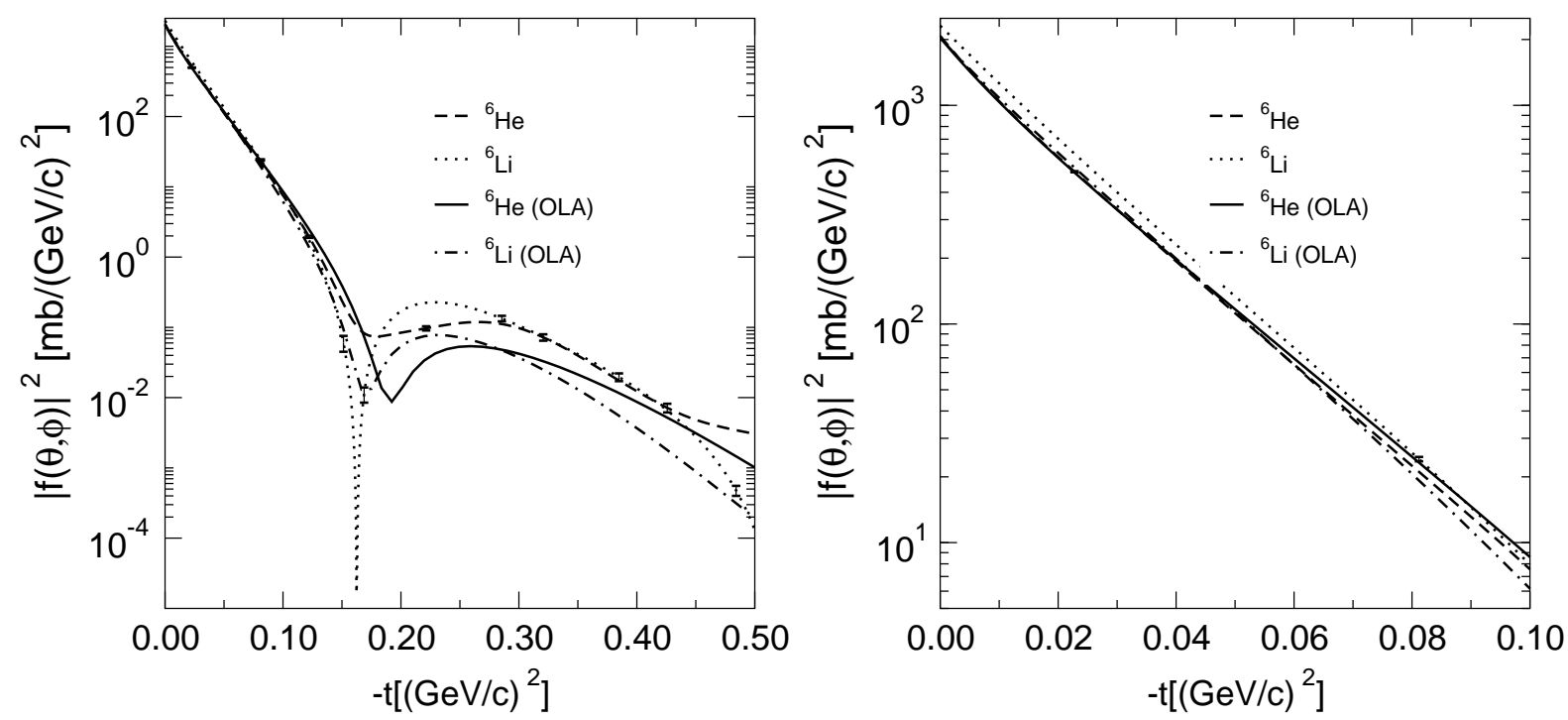

FIG. 5: Comparision of the full and OLA differential cross sections. The parameters are listed in the caption of Fig. 4. The GFMC results are used to calculate the cross sections.

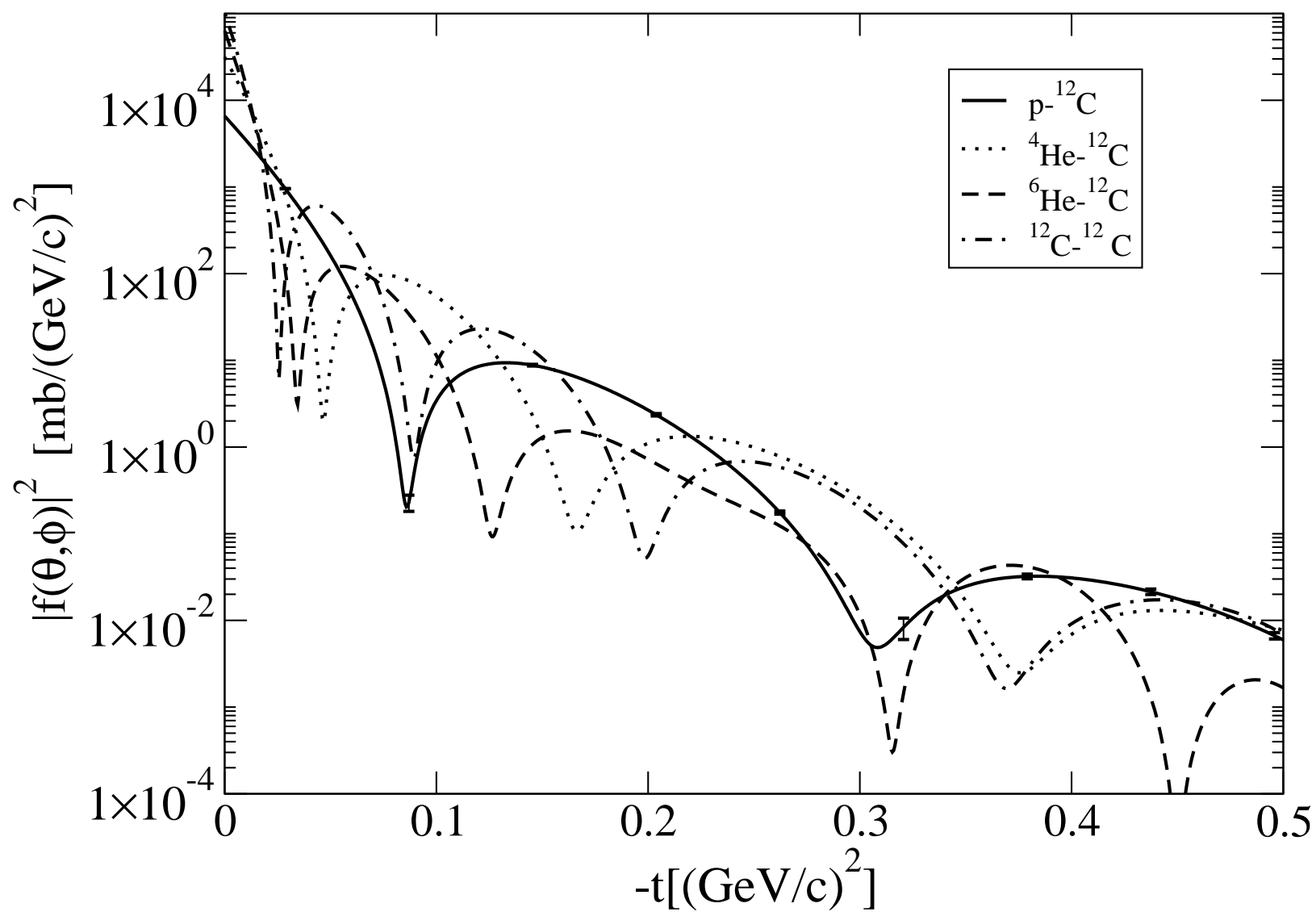

FIG. 6: Differential cross sections versus four-momentum transfer squared for elastic scatterings of $\mathrm{p}, \alpha,{ }^{6} \mathrm{He}$ and ${ }^{12} \mathrm{C}$ on ${ }^{12} \mathrm{C}$ at $0.8 \mathrm{GeV} / A$. 